\title{
Investigation of Potential therapeutic value of Shuanghuanglian Chinese medicine based on network pharmacology for coronavirus pneumonia
}

\section{JIASHENG XU}

The Second Affiliated Hospital of Nanchang University

KAILI LIAO

The Second Affiliated Hospital of Nanchang University

Guanyu Zhang

The Second Affiliated Hospital of Nanchang University

YIRAN LI

The Second Affiliated Hospital of Nanchang University

\section{Weimin Zhou}

the Second Affiliated Hospital of Nanchang University

Jiehua Qiu ( $\nabla$ qiujiehuancu@163.com )

The Second Affiliated Hospital of Nanchang Univesity

\section{Research Article}

Keywords: Traditional Chinese Medicine Compound, Coronavirus Pneumonia, Disease Target, Active Ingredient, Therapeutic Effect

Posted Date: March 25th, 2020

DOl: https://doi.org/10.21203/rs.3.rs-19014/v1

License: (c) (i) This work is licensed under a Creative Commons Attribution 4.0 International License. Read Full License 


\section{Abstract}

Objective: The interaction network between coronavirus pneumonia and Shuanghuanglian was established to explore the potential therapeutic effect of the active ingredients of Shuanghuanglian on coronary virus pneumonia.

Methods: Using the TCMSP database, the effective components of Shuanghuanglian were obtained by screening consistent with oral utilizationखdrug similarity and blood-brain barrier permeability thresholds, and the drug target prediction was performed.The SARS treatment target mining was performed through the GeneCards database, and the two data sets of therapeutic target and drug target were analyzed and the intersection was screened, and the wayne map was drawn.The intersection genes were used as potential therapeutic targets. Cytoscape 3.6 software was used to build a drug active ingredienttherapeutic target interaction network and analyze the active ingredient-therapeutic target point network Degree parameters to find important active ingredients and targets. Using DAVID and String databases to perform GO,KEGG enrichment analysis and protein interaction analysis on the intersection genes to find out the potential signal pathway of Shuanghuanglian against SARS.

Results: 43 effective components against SARS were screened, includingcoptisine MandenolaEthyl

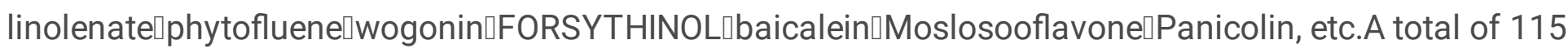
intersection genes with coronavirus pneumonia were screened as important treatment targets: based on the protein interaction network, Shuanghuanglian's therapeutic targets for diseases were significantly enriched in $1763 \mathrm{GO}$ and $133 \mathrm{KEGG}$ signaling pathways; The main action pathways are: responses to steroid hormones and ketones, and development of the reproductive system, responses to lipopolysaccharides, Toxins, responses to bacteria and aging, and effects on epithelial cell proliferation.

Conclusion: The active ingredients in Shuanghuanglian Chinese medicine compound can act on the disease-related target of coronavirus pneumonia and have potential therapeutic effects on coronavirus pneumonia.

\section{Introduction}

Viral pneumonia is a common respiratory disease, but it is still the leading cause of death in young children and the elderly in developing countries. The second leading cause of death was upper respiratory tract infection in 2013, according to The Global Burden of Diseas Study [1]reports, with an agestandardized mortality of $41.7(95 \% \mathrm{Cl} 37.1-44.1)$.And the incidence of pneumonia caused by upper respiratory infection is about $1.5-14$ per 1,000 people per year [2-4] .The incidence of communityacquired pneumonia is U-shaped. It is common in children under 5 years old and adults over 65 years old, and more common in men than in women. In 2008,1.6 million children under 5 years old died of pneumonia [5]. Although Streptococcus pneumoniae is the main pathogen that causes communityacquired pneumonia worldwide $[6,7,8]$ accounting for about $27.3 \%$, but the virus is the most common cause of acute respiratory infections. 
Unfortunately, so far no specific therapies for SARS-CoV, MERS-CoV and other HCoV infections have been found. Symptomatic treatment and supportive treatment are the main means for patients with HCov infection. Although ribavirin and various types of interferon have become the most common treatment attempted by patients with SARS and MERS, The clinical effects are inconsistent when used alone to treat SARS.In vitro studies have shown that the combined use of IFN- $\beta$ can make these two drugs have better antiviral activity, but the clinical efficacy is still controversial (16).At present, the 2019-nCov new coronavirus epidemic outbreak in the world From the current reports in various places, the structure, function.clinical symptoms and disease progression of the emerging coronavirus are extremely similar to SARS-CoV.The current treatment measures for 2019-nCov pneumonia are mostly refered to the treatment of SARS, and 2019-nCov and SARS-CoV have high Sequence homology.Therefore, we take SARS-CoV as the main research object, and it is of great significance to find effective and low-toxicity drugs to treat coronavirus pneumonia.

Shuanghuanglian is a traditional Chinese medicine compound, which has the functions of relieving wind and relieving phlegm, clearing heat and detoxifying, and is widely used in China for fever and cold. It is reported that Shuanghuanglian can inhibit the production of proinflammatory cytokines and chemokines and help fight inflammation and immunity Regulating effect in the rat model of chronic Pseudomonas aeruginosa pneumonia. Shuanghuanglian can increase the lung resistance of rats to chronic Pseudomonas aeruginosa. Shuanghuanglian is also an effective drug for upper respiratory tract infection and acute tonsillitis (17-20). By analyzing the chemical components and the the action genes involved in the compound Chinese herbal medicine Shuanghuanglian and integrate them with viral

Pneumonia related genes to build a drug-gene-disease interaction network. Through the analysis of the interaction network, we predict whether Shuanghuanglian can act on coronavirus pneumonia and treat it.

\section{Materials And Methods}

\subsection{Acquisition of Active Components and Target Genes of Shuanghuanglian}

In this study, the traditional Chinese medicine system pharmacology analysis platform (http://tcmspw.com/tcmsp.php) was used to search for "Hydronic honeysuckle", "Scutellaria baicalensis". "Forsythia" three flavors of traditional Chinese medicine ingredients as Herb name, using oral bioavailability (OB ). Drug-like properties $(\mathrm{DL})$ and cell penetration rate assessment (caco-2) are used as screening indicators, and $\mathrm{OB} \geq 30 \%, \mathrm{DL} \geq 0.18$, and caco- $2 \geq 0.4$ are selected as the active ingredients that meet the conditions. The activity of Shuanghuanglian was obtained through the TCMSP database. The target corresponding to the component is entered into the uniprot database (https://www.uniprot.org/) to obtain the standard gene name of the drug target.

1.2 Screening of target genes for severe acute respiratory syndrome (Severe Acute Respiratory Syndromes, SARS) 
Search the Gene Cards (https://www.genecards.org) database for "Severe Acute Respiratory Syndromes" as a keyword to search for target genes related to severe acute respiratory syndrome.

\subsection{Core target screening}

Use Venny2.1 (http://bioinfogp.cnb.csic.es/tools/venny/index.html) online tool to draw a Wayne diagram of Shuanghuanglian related active ingredient target and Sars target to obtain its intersection target. Investigate the targeting effect of the active ingredients of Shuanghuanglian on Sars target.

\subsection{Component-target network construction and topology analysis}

The compound-target interaction network and the Shuanghuanglian pharmacological component-target interaction network were constructed by Cytoscape 3.6 software. Traditional Chinese medicine components or targets are represented as nodes in the network, and their relationship is represented as edges.Using Net-workAnalyzer computational node degree (Degree) parameters in Cytoscape3.6 software to evaluate the importance of medicinal ingredients and targets.

\subsection{Construction of Shuanghuanglian target protein interaction (PPI) network}

Enter the obtained intersection target genes into the String database (http://string-db.org), set the species to "Homo sapiens", select the data with a confidence level higher than 0.7 , and obtain the protein interaction information corresponding to the intersection genes, and use the online tool to draw the protein mutual aid network diagram.

\subsection{Enrichment analysis and pathway analysis}

The obtained intersection genes were fed into the DAVID database (https://david.ncifcrf.gov/) for gene ontology (GO) biological process enrichment analysis and KEGG pathway enrichment analysis $(p<0.05)$. GO and KEGG are displayed in the form of bubble charts through R-studio software.

\section{Results}

2.1 Analysis of Shuanghuanglian Active Ingredients and Their Targets

Traditional Chinese Medicine System Pharmacology Database and Analysis Platform (TCMSP) (http://tcmspw.com/tcmsp.php) is a network pharmacology database that contains information on traditional Chinese medicine ingredients and their corresponding corresponding targets. This database contains the absorption of traditional Chinese medicine ingredients distribution, metabolism, and key parameters of excretion, including drug similarity (DL), oral bioavailability (OB), blood-brain barrier transmission rate (BBB), etc. This study uses the TCMSP analysis platform to identify "Honeysuckle" , "Scutellaria baicalensis"," Forsythia "three Chinese medicine ingredients were searched as Herb name, using oral bioavailability ( $\mathrm{OB})$, Drug-likeness (DL) and cell penetration rate assessment (caco-2) as screening indicators, select $\mathrm{OB} \geq 30 \%, \mathrm{DL} \geq 0.18$, caco- $2 \geq 0.4$ as eligible active ingredients. The target 
corresponding to the active ingredient of Shuanghuanglian is obtained through the TCMSP database and entered into the uniprot database (https://www.uniprot.org/) to obtain the standard gene name of drug target.45 active molecules are now analyzed, including MandenolaEthyl linolenate, $\mathrm{b}$ beta-carotene[ ZINC03978781®5-hydroxy-7-methoxy-2-(3,4,5-trimethoxyphenyl)chromone beta-sitosterol $[$ Stigmasterol $\square$

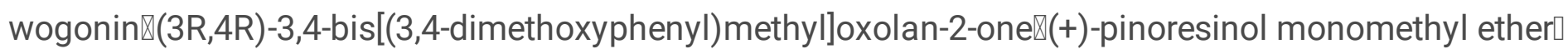
ACon1_001697区(+)-pinoresinol monomethyl ether-4-D-beta-glucoside_qt『3beta-Acetyl-20,25-

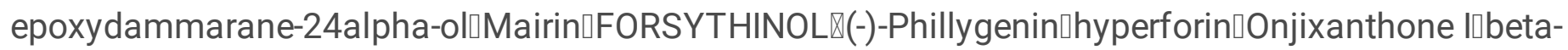

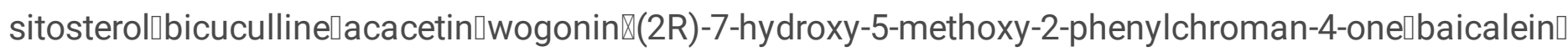

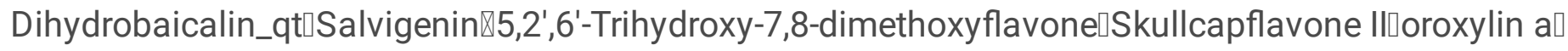

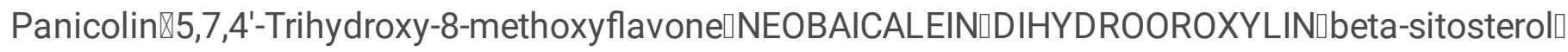
sitosterol $\varangle 5,2$-Dihydroxy-6,7,8-trimethoxyflavone $\llbracket S t i g m a s t e r o l[c o p t i s i n e \llbracket b i s[(2 S)$-2-ethylhexyl] benzene-

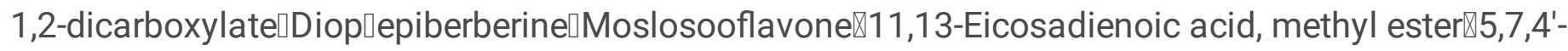
trihydroxy-6-methoxyflavanone『rivularin $\rrbracket$

According to the chemical composition information of Shuanghuanglian, its target targets were predicted, and 122 standard gene names corresponding to molecular targets were obtained, including

ACHE $\llbracket A D H 1 C \square A D R A 1 A \square A D R A 1 B \square A D R A 1 D \square A D R A 2 A \square A D R B 1 \square A D R B 2 \square A H R \square A H S A 1 \square A K R 1 B 1 \square A K T 1 \square A L B \square$

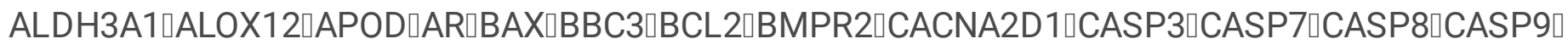

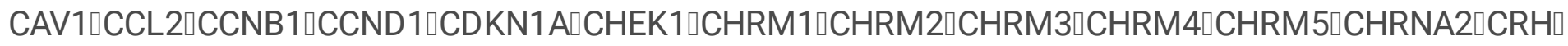

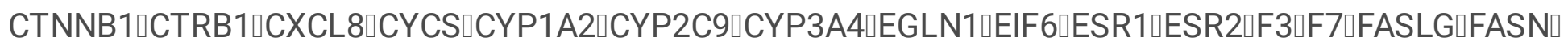

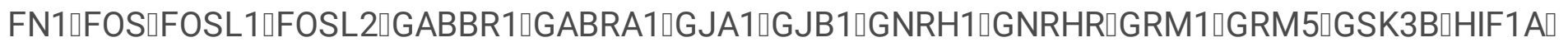

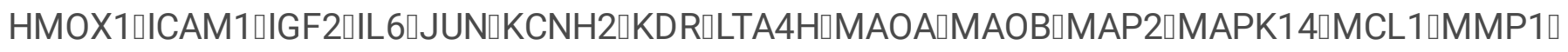

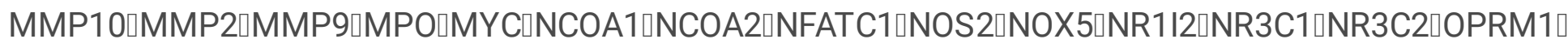

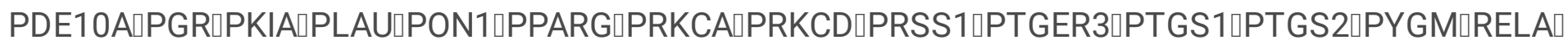

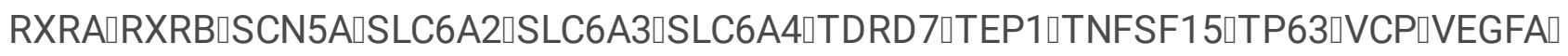

2.2 Screening of target genes for severe acute respiratory syndrome (Severe Acute Respiratory Syndromes, Sars)

The GeneCards (https://www.genecards.org) database was used to search for target genes related to severe acute respiratory syndrome using "Severe Acute Respiratory Syndromes" as keywords, and 7820 gene targets were obtained.

2.3 Core target screening

Using Venny2.1 (http://bioinfogp.cnb.csic.es/tools/venny/index.html) online tool to draw a Venn diagram of Shuanghuanglian related active ingredient target and Sars target to obtain the intersection target 115(Figure 1), including ACHE\ ADH1C $\triangle A D R A 1 A \rrbracket A D R A 1 B \square A D R A 1 D \square A D R A 2 A \rrbracket A D R B 1 \square A D R B 2 \square A H R \square$

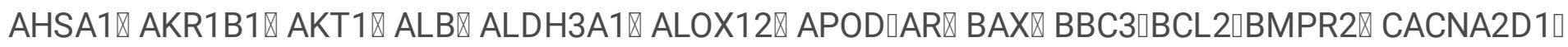

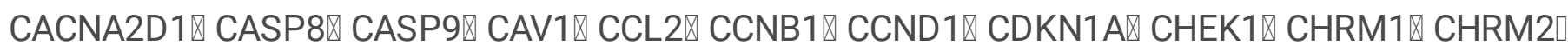

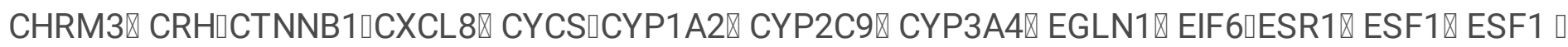




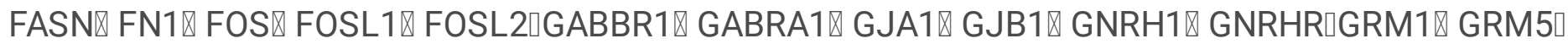

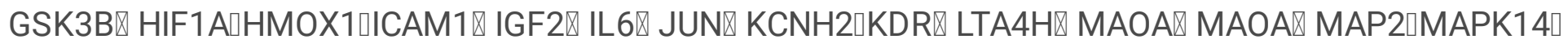

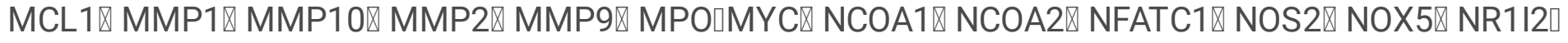

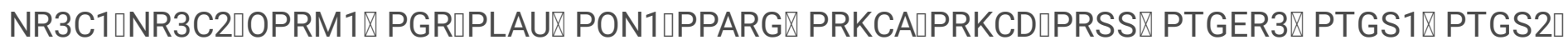

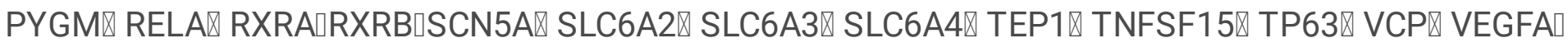

\subsection{Component-target network construction and topology analysis}

Compound-target interaction network and Shuanghuanglian medicinal ingredient-target interaction network were constructed by Cytoscape 3.6 software (see Figure 2). Traditional Chinese medicine ingredients or targets are represented as nodes in the network, a total of 159 between them. The connections are represented as edges, a total of 599.

2.5 Construction of Shuanghuanglian target protein interaction (PPI) network

Enter the obtained intersection target genes into the String database (http://string-db.org), set the species to "Homo sapiens", select the data with a confidence level higher than 0.7 , and obtain the protein interaction information corresponding to the intersection genes and 5 cluster interaction networks(Figure 3). Cluster 1 has 38 nodes, including PLAU, MCL1, MMP1, ESR1, CCL2, PGR, GJA1, IL6, CTNNB1, PPARG, CXCL8, FASLG, RELA, CYCS, CASP9, FOS, JUN, MMP9, CASP3, CASP8, ICAM1, ALB, FN1, HIF1A, AKT1, HMOX1, KDR, CCNB1, VEGFA, CCND1, CDKN1A, MMP2, AR, MPO, MAPK14, PTGS2, IGF2, MYC. A total of 561 interaction lines. Cluster 2 has 8 nodes, including CHRM3, GRM5, CHRM1, GRM1, ADRA1A, ADRA1D, GNRHR, PRKCA, a total of 23 interaction lines. Cluster 3 has 14 nodes, CHRM2, ACHE, CYP2C9, CYP1A2, SLC6A4, GABBR1, OPRM1, PON1, GNRH1, MAOA, MAOB, ADRA2A, ADRA1B, ADRB1, a total of 41 interaction lines. Cluster4 has 6 nodes, CAV1, AHR, ESR2, NR3C1, NOS2, GSK3B, a total of 9 interaction lines. Cluster5 has 3 Node, NR1I2, RXRA, RXRB, a total of 3 interaction lines.

\subsection{Target gene enrichment analysis}

Using the David database to perform gene ontology analysis (Go analysis) on the core targets of sars, a total of 1763 go enrichment analyses were identified, which involved 1,542 biological processes (BP),72 cell compositions (CC), 149 molecular functions (MF). Sorted according to the size of the $P$-value, each selected 10 enriched Go values for visual display (Figure 4A). The top 10 of the biological process (BP), cell composition (CC), and molecular function (MF) in GO analysis were shown in Table 1. We noticed that some biological processes may be related to steroid hormones, ketones, lipids. polysaccharides, toxic substances, bacterial origin molecules and reproductive systems, aging and epithelial cell proliferation were related; Shuanghuanglian's resistance to Sars may be related to the membrane cell composition such as cell membranes and pre-synaptic membranes, which may be related to DNA transcription ,nuclearreceptor. steroid hormone receptors, G-protein-coupled amine receptors, or neurotransmitter receptor activity.

Analysis of target KEGG pathway in acute respiratory distress syndrome 
DAVID database was used to perform KEGG signal pathway enrichment analysis on core targets in the acute respiratory distress syndrome network, and visualized after sorting by $P$ value and Gene ratio(Figure 4B). In the analysis of KEGG signal pathway, the $P$ value and GeneRatio of the top 20 signal pathways are shown in Table 2. The related parameters in the drawn bubble chart are shown in Table 3. The results show that Shuanghuanglian resists respiratory distress syndrome which mainly involves AGERAGE, IL-17, p53, TNF, estrogen,thyroid hormone and other signal pathways, Kaposi's sarcoma-associated herpes virus, human cytomegalovirus, measles virus, HIV, hepatitis $B$, hepatitis $C$ and other virus, viral infections, small cell lung cancer, colorectal cancer and other cancer related pathways and neuroactive ligand-receptor interactions, Apoptosis, Endocrine resistance and other pathways.

\section{Discussion}

In the past decade or so, several previously undiscovered and confirmed viruses have appeared around the world, which cause respiratory diseases that are highly contagious and highly lethal. Especially new coronaviruses such as: SARS-CoV, H1N1-CoV, H5N1-CoV, MERS-CoV can cause tens of thousands of people worldwide infect, hundreds of people die, and because of its infectiousness, whenever pneumonia caused by this virus breaks out, in order to stop the infection sources and routes of transmission inevitably take various isolation measures, which often lead to people's panic and the abnormal functioning of society, causing inestimable losses. The histopathological changes of viral pneumonia vary, and may be related to viral infection and complications. Usually, interstitial pneumonia with lymphocytic infiltration can be seen in viral pneumonia [21]. And fatal cases of Sars and H5N1 were found to be characterized by alveolar damage, peeling of lung cells, edema and the formation of transparent membranes [22, 23]. Recently, a new virus 2019-nCoV was discovered in Wuhan, China. It belongs to the Coronaviridae family as well as the SARS virus and the MERS virus. At present, the new virus pneumonia caused by it is becoming increasingly infected and deadly for many people. Human coronavirus is considered to be an important pathogen causing infections in children, the elderly and immunocompromised patients, including upper and lower respiratory tract infections and acute respiratory distress syndrome [24]. Between 2002 and 2003, a global outbreak caused by SARS virus, which has infected 8089 people worldwide and killed 916 people in just a few months (25). Viral pneumonia caused by SARS virus, patients usually start acutely, have flu-like symptoms, dyspnea, and recurrent or persistent fever. The imaging characteristics of SARS are similar to those of other community-acquired pneumonias.

At first, chest X-rays are normal, and then quickly develop into nodular shadows. Large patchy shadows or large airspace consolidation shadows are mainly concentrated in the lower lung and Involving surrounding lung tissues .MERS Coronavirus is a causative agent of the Middle East Respiratory Syndrome. During 2012-2014, there was an outbreak of MERS Coronavirus in Saudi Arabia, with a total mortality rate of $35 \%-44 \%$ (26). In March,2015, a large-scale MERS coronavirus pandemic occurred in South Korea, with 186 patients diagnosed and 38 deaths. The clinical symptoms of patients with Middle East Respiratory Syndrome are similar to those involving fever, cough, dyspnea and pneumonia. Infection may rapidly develop into acute respiratory distress syndrome, multiple organ failure, and death. 
Compared with SARS, MERS progresses to respiratory failure and causes is faster and causes acute kidney damage (27). What is more frightening is for new coronaviruses like SARS-CoV. MERS-CoV have so far lacked effective clinical antiviral drugs. These patients mainly received supportive treatment.Although during the SARS-MERS outbreak, ribavirin and inflammatory corticosteroids or IFNa with immunoglobulin.Thymosin in combination therapy (28-34), but these treatments have not been tested in clinical trials, their efficacy is difficult to assess and retrospective analysis has not draw an effective treatment combination. In addition, according to previous reports, it is unknown whether ribavirin has a greater benefit to the disease than it does to the body when it is used alone. However,IFN was proved to be effective against MERS-CoV (35-37) in vitro, and demonstrated in the MERS rhesus (38) model, the combined use of IFNa and ribovirus can alleviate disease, but it also brings such things as: fatigue, depression, anemia and other side effects. These greatly limit the use of IFNa as a first-line drug (39-41). Therefore, it is particularly important to find drugs that have inhibitory or therapeutic effects on such viral pneumonia and have fewer side effects. Since Professor Tu Youyou won the Nobel Prize in Physiology or Medicine for discovering the therapeutic effect of artemisinin on malaria in 2015, the effect of Chinese medicine on the disease has quickly attracted the attention of the medical community $(42,43)$. In addition to artemisinin many traditional Chinese medicines have been shown to play an important role in the disease, for example: curcumin in turmeric has been shown to inhibit ovarian cancer, skin cancer, colorectal cancer, head and neck squamous cell carcinoma, and gastric cancer (4448). Ginsenosides extracted from Fax Chen, Panax ginseng, and cinnamon (Cinnamomum cassia Presl) can inhibit cell proliferation, migration, angiogenesis, and anti-drug resistance (49-57) Plays anti-cancer effects in colorectal cancer, breast cancer, liver cancer and lung cancer (32-35). Emodin can be isolated from rhubarb palm, Polygonum cuspidatum, Polygonum multiflorum and Cassia seed, which has antiinflammatory, anti-oxidant, prevent hepatic fat accumulation and DNA damage (58-64) has been shown to slow nasopharyngeal cancer, gallbladder cancer, lung cancer, liver cancer, colorectal cancer, oral cancer, ovarian cancer, bladder cancer, prostate cancer, breast cancer, gastric and pancreatic cancer (65-77).

Shuanghuanglian is a traditional Chinese medicine compound consisting of honeysuckle, scutellaria baicalensis and forsythia, which has been widely used in China for a long time. Shuanghuanglian injection can inhibit the inflammation associated with viral encephalitis, and it has been reported that Shuanghuanglian can play anti-inflammatory and antioxidant roles in alveolar macrophages of rats. Shuanghuanglian injection can prevent H5N1 virus infection by inhibiting viral replication and reducing lung injury and can effectively exert anti-HAdV virus effects by inhibiting virus penetration (78-79). We analyze the effective compounds in Shuanghuanglian traditional Chinese medicine compound, explore the proteins and corresponding genes that they act on, and compare the genes that act with SARS-related genes, and find that these two types of genes have 151 common gene action targets. Then we used Shuanghuanglian active ingredients and disease target genes to build a drug-disease-gene interaction network map. We use the intersection target genes as potential target genes for Shuanghuanglian treatment of coronavirus pneumonia. These target genes were enriched by gene function and action pathways,and the results were found: the main pathways of action are the response to steroid hormones and ketones, the development of the reproductive system, the response to lipopolysaccharides. Toxins, 
the response to bacteria and aging, and the effect on epithelial cell proliferation. Coronavirus often causes viral pneumonia, destroys normal alveolar tissue, proliferates fibroblasts and aggregates a large number of extracellular matrices, resulting in pulmonary fibrosis, resulting in severe loss of lung function,Corticosteroids, anti-inflammatory and supportive treatments are currently for diseases caused by the kind of coronavirus like SARS-CoV, but it is well known that large doses and prolonged use of corticosteroids can cause obesity, immune system disorders, corticosteroid signs, electrolyte disorders, gastrointestinal ulcers, and severe necrosis of the femoral head. According to our research, the active ingredients of Shuanghuanglian traditional Chinese medicine compound have an effect on the proliferation of epithelial cells and bacteria and aging. It also has an effect on the response of steroid hormones, lipopolysaccharides and toxins, so the compound Chinese herbal medicine Shuanghuanglian may play an important role in the treatment of diseases caused by coronavirus. On the other hand, we found that the effective ingredients of Shuanghuanglian. The main pathways enriched for target genes are: cytomegalovirus infection, immunodeficiency virus infection, apoptosis, endocrine resistance, IL-17 signaling pathway, p53 signaling pathway, TNF signaling pathway.Viral infection, low immune function, and lung tissue, fibrosis is a feature of diseases caused by, for example, SARS-CoV.

\section{Conclusion}

The signal pathways enriched in this study are closely related to the diseases caused by coronavirus, and it suggests the feasibility of Shuanghuanglian traditional Chinese medicine compound for the treatment of diseases caused by coronavirus such as SARS. The active ingredients in Shuanghuanglian Chinese medicine compound can act on the disease-related target of coronavirus pneumonia and have potential therapeutic effects on coronavirus pneumonia.

\section{Declarations}

\section{Ethical approval and Consent to participate :}

This article does not contain any studies with animals or human performed by any of the authors. All methods are carried out in accordance with relevant guidelines and regulations.

Consent for publication $\bigotimes$ Not applicable.

\section{Availability of supporting data:}

All datas are available. Please contact us to access if it is needed.

Competing interests: There is no conflict of interests in this study: all authors declare that they have no conflict of interest.

\section{Funding}


This study is supported by the national natural science foundation of China [81860095]; the Natural Science Foundation of Jiangxi Province [20171BAB205005] and the Health office of the Natural Science Foundation of Jiangxi Province [20175218].

\section{Authors' contributions}

Jiasheng Xu and Kaili Liao analysed most of the data, and wrote the initial draft of the paper.

Guanyu Zhang and Yiran Li contributed to refining the ideas, carrying out additional analyses and finalizing this paper.

Weimin Zhou and Jiehua Qiu contributed the central idea.

\section{Acknowledgements $]$}

Not applicable.

\section{Authors' information}

Jiasheng Xu

Institution: Department of Vascular Surgery, The Second Affiliated Hospital of Nanchang University, Nanchang 330006, Jiangxi, China.

E-mail:1821286450@qq.com

Kaili Liao

Institution: Department of Clinical Laboratory, the Second Affiliated Hospital of Nanchang University, No. 1 Minde Road, Nanchang, Jiangzi 330006, China

E-mail:1192784983@qq.com

Guanyu Zhang

Institution: Department of Vascular Surgery, The Second Affiliated Hospital of Nanchang University, Nanchang 330006, Jiangxi, China.

E-mail:825761553@qq.com

Yiran Li

Institution: Department of Vascular Surgery, The Second Affiliated Hospital of Nanchang University, Nanchang 330006, Jiangxi, China.

E-mail:895600186@qq.com 
Weimin Zhou

Institution: Department of Vascular Surgery, The Second Affiliated Hospital of Nanchang University, Nanchang 330006, Jiangxi, China.

E-mail:jesonxudoctor@163.com

Jiehua Qiu

Institution: Department of Vascular Surgery, The Second Affiliated Hospital of Nanchang University, Nanchang 330006, Jiangxi, China.

E-mail: qiujiehuancu@163.com

\section{References}

[1]GBD 2013 Mortality and Causes of Death Collaborators. Global, regional, and national age-sex specifi c all-cause and cause-specifi c mortality for 240 causes of death, 1990-2013:

[2]a systematic analysis for the Global Burden of Disease Study 2013. Lancet 2015; 385: 117-71.

[3]Millett ER, Quint JK, Smeeth L, Daniel RM, Thomas SL. Incidence of community-acquired lower respiratory tract infections and pneumonia among older adults in the United Kingdom: a populationbased study. PLoS One 2013; 8: e75131.

[4]Ochoa-Gondar O, Vila-Córcoles A, de Diego C, et al, for the EVAN-65 Study Group. The burden of community-acquired pneumonia in the elderly: the Spanish EVAN-65 study. BMC Public Health 2008; 8: 222.

[5]File TM Jr, Marrie TJ. Burden of community-acquired pneumonia in North American adults. Postgrad Med 2010; 122: 130-41

[6]Black RE, Cousens S, Johnson HL, et al, for the Child Health Epidemiology Reference Group of WHO and UNICEF. Global, regional, and national causes of child mortality in 2008: a systematic analysis. Lancet 2010; 375: 1969-87.

[7]Welte T, Torres A, Nathwani D. Clinical and economic burden of community-acquired pneumonia among adults in Europe. Thorax 2012; 67: 71-79.

[8]Drijkoningen JJ, Rohde GG. Pneumococcal infection in adults: burden of disease. Clin Microbiol Infect 2014; 20 (suppl 5): 45-51.

[9]Efferth T. From ancient herb to modern drug: artemisia annua and artemisinin for cancer therapy. Semin Cancer Biol. 2017;46:65-83. doi: 10.1016/j.semcancer.2017.02.009. 
[10]Chen, Xin et al. 2002. "Effects of Shuanghuanglian and Qingkailing, Two Multi-Components of Traditional Chinese Medicinal Preparations, on Human Leukocyte Function." Life sciences 70(24): 28972913.

[11]Li, Wei. 2002. "[The curative effect observation of shuanghuanglian and penicillin on acute tonsillitis]." Lin chuang er bi yan hou ke za zhi = Journal of clinical otorhinolaryngology 16(9): 475-76.

[12]Song, Z J et al. 2000. "Effects of Radix Angelicae Sinensis and Shuanghuanglian on a Rat Model of Chronic Pseudomonas Aeruginosa Pneumonia." Chinese medical sciences journal = Chung-kuo i hsueh k'o hsueh tsa chih 15(2): 83-88.

[13]Zhang, Hongwei et al. 2013. "Chinese Medicine Injection Shuanghuanglian for Treatment of Acute Upper Respiratory Tract Infection: A Systematic Review of Randomized Controlled Trials." Evidence-based complementary and alternative medicine: eCAM 2013: 987326.

[14]Howard LS, Sillis M, Pasteur MC, Kamath AV, Harrison BD. Microbiological profi le of communityacquired pneumonia in adults over the last 20 years. J Infect 2005; 50: 107-13.

[15]do Carmo Debur M, Raboni SM, Flizikowski FBZ, et al. Immunohistochemical assessment of respiratory viruses in necropsy samples from lethal and non-pandemic seasonal respiratory infections. $J$ Clin Pathol 2010; 63: 930-34.

[16]Nicholls JM, Poon LLM, Lee KC, et al. Lung pathology of fatal severe acute respiratory syndrome. Lancet 2003; 361: 1773-78.

[17]Korteweg C, Jiang G. Pathology, molecular biology, and pathogenesis of avian infl uenza A (H5N1) infection in humans. Am J Pathol 2008; 172: 1155-70.

[18]World Health Organization WHO Guidelines for the Global Surveillance of Severe Acute Respiratory Syndrome (SARS) [(accessed on 8 October 2018)];2004 Oct; Updated Recommendations. Available online: http://www.who.int/mediacentre/factsheets/mers-cov/en/

[19]Mackay IM, Arden KE. MERS coronavirus: diagnostics, epidemiology and transmission. Virol J 2015;12(1):222.

[20]von Grotthuss M, Wyrwicz LS, Rychlewski L. mRNA cap-1 methyltransferase in the SARS genome. Cell 2003; 113: 701-2.

[21]Lee, N. et al. A major outbreak of severe acute respiratory syndrome in Hong Kong. N. Engl. J. Med. 348, 1986-1994 (2003).

[22]Booth, C. M. et al. Clinical features and short-term outcomes of 144 patients with SARS in the greater Toronto area. JAMA 289, 2801-2809 (2003). 
[23]Poutanen, S. M. et al. Identification of severe acute respiratory syndrome in Canada. N. Engl. J. Med. 348, 1995-2005 (2003).

[24]So, L. K. et al. Development of a standard treatment protocol for severe acute respiratory syndrome. Lancet 361, 1615-1617 (2003).

[25]Tsang, K. W. et al. A cluster of cases of severe acute respiratory syndrome in Hong Kong. N. Engl. J. Med. 348, 1977-1985 (2003).

[26]Loutfy, M. R. et al. Interferon alfacon-1 plus corticosteroids in severe acute respiratory syndrome: a preliminary study. JAMA 290, 3222-3228 (2003).

[27]Zhao, Z. et al. Description and clinical treatment of an early outbreak of severe acute respiratory syndrome (SARS) in Guangzhou, PR China. J. Med. Microbiol. 52, 715-720 (2003).

[28]Chan, J. F. et al. Broad-spectrum antivirals for the emerging Middle East respiratory syndrome coronavirus. J. Infect. 67, 606-616 (2013).

[29]Falzarano, D. et al. Inhibition of novel $\beta$ coronavirus replication by a combination of interferon- $a 2 b$ and ribavirin. Sci. Rep. 3, 1686 (2013).

[30]Hart, B. J. et al. Interferon- $\beta$ and mycophenolic acid are potent inhibitors of Middle East respiratory syndrome coronavirus in cell-based assays. J. Gen. Virol. 95, 571-577 (2014).

[31]Falzarano, D. et al. Treatment with interferon-a2b and ribavirin improves outcome in MERS-CoVinfected rhesus macaques. Nat. Med. 19, 1313-1317 (2013). The first application of a potential treatment option for MERS through the repurposing of IFNa2b and ribavirin in a non-human primate model.

[32]Morgenstern, B., Michaelis, M., Baer, P. C., Doerr, H. W. \& Cinatl, J. Ribavirin and interferon- $\beta$ synergistically inhibit SARS-associated coronavirus replication in animal and human cell lines. Biochem. Biophys. Res. Commun. 326, 905-908 (2005).

[33]Falzarano, D. et al. Inhibition of novel $\beta$ coronavirus replication by a combination of interferon- $a 2 b$ and ribavirin. Sci. Rep. 3, 1686 (2013).

[34] Hart, B. J. et al. Interferon- $\beta$ and mycophenolic acid are potent inhibitors of Middle East respiratory syndrome coronavirus in cell-based assays. J. Gen. Virol. 95, 571-577 (2014).

[35]Gong Y., Huang X., Wang D., Li M., Liu Z. (2017). Triptolide protects bone against destruction by targeting RANKL-mediated ERK/AKT signalling pathway in the collagen-induced rheumatoid arthritis. Biomed. Res. 28 4111-4116. 
[36]Efferth T. From ancient herb to modern drug: artemisia annua and artemisinin for cancer therapy. Semin Cancer Biol. 2017;46:65-83. doi: 10.1016/j.semcancer.2017.02.009.

[37]Efferth T, Zacchino S, Georgiev MI, Liu L, Wagner H, Panossian AJ, et al. Nobel prize for artemisinin brings phytotherapy into the spotlight. Phytomedicine. 2015;22(13):1-4. doi:

10.1016/j.phymed.2015.10.003.

[38]Cai YY, Lin WP, Li AP, Xu JY. Combined effects of curcumin and triptolide on an ovarian cancer cell line. Asian Pac J Cancer Prev. 2013;14(7):4267-4271. doi: 10.7314/APJCP.2013.14.7.4267.

[39]Jose A, Labala S, Venuganti VVK. Co-delivery of curcumin and STAT3 siRNA using deformable cationic liposomes to treat skin cancer. J Drug Target.

[40]Ravindranathan P, Pasham D, Balaji U, Cardenas J, Gu JH, Toden S, et al. A combination of curcumin and oligomeric proanthocyanidins offer superior anti-tumorigenic properties in colorectal cancer.

[41]Sivanantham B, Sethuraman S, Krishnan UM. Combinatorial effects of curcumin with an antineoplastic agent on head and neck squamous cell carcinoma through the regulation of EGFR-ERK1/2 and apoptotic signaling pathways. Acs Comb Sci. 2016;18(1):22-35. doi: 10.1021/acscombsci.5b00043.

[42]Zou P, Zhang JR, Xia YQ, Kanchana K, Guo GL, Chen WB, et al. ROS generation mediates the anticancer effects of WZ35 via activating JNK and ER stress apoptotic pathways in gastric cancer. Oncotarget. 2015;6(8):5860-5876. doi: 10.18632/oncotarget.3333.

[43]Zhang J, Zhou F, Wu X, Zhang X, Chen Y, Zha BS, et al. Cellular pharmacokinetic mechanisms of adriamycin resistance and its modulation by 20(S)-ginsenoside Rh2 in MCF-7/Adr cells. Br J Pharmacol. 2012;165(1):120-134. doi: 10.1111/j.1476-5381.2011.01505.x.

[44]Lu Z, Xu H, Yu X, Wang Y, Huang L, Jin X, et al. 20(S)-Protopanaxadiol induces apoptosis in human hepatoblastoma HepG2 cells by downregulating the protein kinase B signaling pathway. Exp Ther Med. 2018;15(2):1277-1284.

[45]Liu Y, Fan D. Ginsenoside Rg5 induces apoptosis and autophagy via the inhibition of the PI3K/Akt pathway against breast cancer in a mouse model. Food Funct. 2018;9:5513-5527. doi:

10.1039/C8FO01122B.

[46]Wu Q, Deng J, Fan D, Duan Z, Zhu C, Fu R, et al. Ginsenoside Rh4 induces apoptosis and autophagic cell death through activation of the ROS/JNK/p53 pathway in colorectal cancer cells. Biochem Pharmacol. 2018;148:64-74. doi: 10.1016/j.bcp.2017.12.004.

[47]Leem DG, Shin JS, Kim KT, Choi SY, Lee MH, Lee KT. Dammarane-type triterpene ginsenoside-Rg18 inhibits human non-small cell lung cancer A549 cell proliferation via G1 phase arrest. Oncol Lett. 2018;15(4):6043-6049. 
[48]Zeng D, Wang J, Kong P, Chang C, Li J, Li J. Ginsenoside Rg3 inhibits HIF-1a and VEGF expression in patient with acute leukemia via inhibiting the activation of PI3K/Akt and ERK1/2 pathways. Int J Clin Exp Pathol. 2014;7(5):2172-2178.

[49]Wang L, Gao S, Jiang W, Luo C, Xu M, Bohlin L, et al. Antioxidative dietary compounds modulate gene expression associated with apoptosis, DNA repair, inhibition of cell proliferation and migration. Int J Mol Sci. 2014;15(9):16226-16245. doi: 10.3390/ijms150916226.

[50]Kim YJ, Choi WI, Jeon BN, Choi KC, Kim K, Kim TJ, et al. Stereospecific effects of ginsenoside 20-Rg3 inhibits TGF-beta1-induced epithelial-mesenchymal transition and suppresses lung cancer migration, invasion and anoikis resistance. Toxicology. 2014;322:23-33. doi: 10.1016/j.tox.2014.04.002.

[51]Yang J, Yuan D, Xing T, Su H, Zhang S, Wen J, et al. Ginsenoside Rh2 inhibiting HCT116 colon cancer cell proliferation through blocking PDZ-binding kinase/T-LAK cell-originated protein kinase. J Ginseng Res. 2016;40(4):400-408. doi: 10.1016/j.jgr.2016.03.007.

[52] He NW, Zhao Y, Guo L, Shang J, Yang XB. Antioxidant, antiproliferative, and pro-apoptotic activities of a saponin extract derived from the roots of Panax notoginseng (Burk.) F.H. Chen. J Med Food. 2012;15(4):350-359. doi: 10.1089/jmf.2011.1801.

[53]Lu M, Fei Z, Zhang G. Synergistic anticancer activity of 20(S)-Ginsenoside Rg3 and Sorafenib in hepatocellular carcinoma by modulating PTEN/Akt signaling pathway. Biomed Pharmacother. 2018;97:1282-1288. doi: 10.1016/j.biopha.2017.11.006.

[54]Wang X, Sun YY, Zhao C, Qu FZ, Zhao YQ. 12-Chloracetyl-PPD, a novel dammarane derivative, shows anti-cancer activity via delay the progression of cell cycle $\mathrm{G} 2 / \mathrm{M}$ phase and reactive oxygen speciesmediate cell apoptosis. Eur J Pharmacol. 2017;798:49-56. doi: 10.1016/j.ejphar.2016.12.027.

[55]Lee IS, Uh I, Kim KS, Kim KH, Park J, Kim Y, et al. Anti-inflammatory effects of ginsenoside Rg3 via NFkappaB pathway in A549 cells and human asthmatic lung tissue. J Immunol Res. 2016;2016:7521601.

[56]Chen G, Zhang J, Zhang H, Xiao Y, Kao X, Liu Y, et al. Anti-inflammatory effect of emodin on lipopolysaccharide-induced keratitis in Wistar rats. Int J Clin Exp Med. 2015;8(8):12382-12389. [PMC free article] [PubMed] [Google Scholar]

[57]Li Y, Xiong W, Yang J, Zhong J, Zhang L, Zheng J, et al. Attenuation of inflammation by emodin in lipopolysaccharide-induced acute kidney injury via inhibition of toll-like receptor 2 signal pathway. Iran J Kidney Dis. 2015;9(3):202-208. [PubMed] [Google Scholar]

[58]Chen GL, Zhang JJ, Kao X, Wei LW, Liu ZY. Emodin ameliorates lipopolysaccharides-induced corneal inflammation in rats. Int J Ophthalmol. 2015;8(4):665-669.

[59]Park SY, Jin ML, Ko MJ, Park G, Choi YW. Anti-neuroinflammatory effect of emodin in LPS-stimulated microglia: involvement of AMPK/Nrf2 activation. Neurochem Res. 2016;41(11):2981-2992. doi: 
[60]Yao WY, Zhou YF, Qian AH, Zhang YP, Qiao MM, Zhai ZK, et al. Emodin has a protective effect in cases of severe acute pancreatitis via inhibition of nuclear factor-kappa B activation resulting in antioxidation. Mol Med Rep. 2015;11(2):1416-1420. doi: 10.3892/mmr.2014.2789.

[61]Alisi A, Pastore A, Ceccarelli S, Panera N, Gnani D, Bruscalupi G, et al. Emodin prevents intrahepatic fat accumulation, inflammation and redox status imbalance during diet-induced hepatosteatosis in rats. Int J Mol Sci. 2012;13(2):2276-2289. doi: 10.3390/ijms13022276.

[62]Sharma R, Tiku AB. Emodin, an anthraquinone derivative, protects against gamma radiation-induced toxicity by inhibiting DNA damage and oxidative stress. Int J Radiat Biol. 2014;90(4):275-283. doi: 10.3109/09553002.2014.884292.

[63]Ma L, Yang Y, Yin Z, Liu M, Wang L, Chen L, et al. Emodin suppresses the nasopharyngeal carcinoma cells by targeting the chloride channels. Biomed Pharmacother. 2017;90:615-625. doi: 10.1016/j.biopha.2017.03.088.

[64]Li XX, Dong Y, Wang W, Wang HL, Chen YY, Shi GY, et al. Emodin as an effective agent in targeting cancer stem-like side population cells of gallbladder carcinoma. Stem Cells Develop. 2013;22(4):554566. doi: $10.1089 /$ scd.2011.0709.

[65]Haque E, Kamil M, Irfan S, Sheikh S, Hasan A, Nazir A, et al. Blocking mutation independent p53 aggregation by emodin modulates autophagic cell death pathway in lung cancer. Int J Biochem Cell Biol. 2018;96:90-95. doi: 10.1016/j.biocel.2018.01.014.

[66]Zhang X, Chen Y, Zhang T, Zhang Y. Inhibitory effect of emodin on human hepatoma cell line SMMC7721 and its mechanism. Afr Health Sci. 2015;15(1):97-100. doi: 10.4314/ahs.v15i1.13.

[67]Ma YS, Weng SW, Lin MW, Lu CC, Chiang JH, Yang JS, et al. Antitumor effects of emodin on LS1034 human colon cancer cells in vitro and in vivo: roles of apoptotic cell death and LS1034 tumor xenografts model. Food Chem Toxicol. 2012;50(5):1271-1278. doi: 10.1016/j.fct.2012.01.033.

[68]Manimaran A, Buddhan R, Manoharan S. Emodin downregulates cell proliferation markers during DMBA induced oral carcinogenesis in Golden Syrian Hamsters. Afr J Tradit Complement Altern Med. 2017;14(2):83-91. doi: 10.21010/ajtcam.v14i2.10

[69]Lu J, Xu Y, Zhao Z, Ke X, Wei X, Kang J, et al. Emodin suppresses proliferation, migration and invasion in ovarian cancer cells by down regulating ILK in vitro and in vivo. OncoTargets Ther. 2017;10:35793589. doi: 10.2147/OTT.S138217.

[70]Cha TL, Chuang MJ, Tang SH, Wu ST, Sun KH, Chen TT, et al. Emodin modulates epigenetic modifications and suppresses bladder carcinoma cell growth. Mol Carcinog. 2015;54(3):167-177. doi: 10.1002/mc.22084. 
[71]Deng G, Ju X, Meng Q, Yu ZJ, Ma LB. Emodin inhibits the proliferation of PC3 prostate cancer cells in vitro via the Notch signaling pathway. Mol Med Rep. 2015;12(3):4427-4433. doi:

10.3892/mmr.2015.3923.

[72]Sun Y, Wang X, Zhou Q, Lu Y, Zhang H, Chen Q, et al. Inhibitory effect of emodin on migration, invasion and metastasis of human breast cancer MDA-MB-231 cells in vitro and in vivo. Oncol Rep. 2015;33(1):338-346. doi: 10.3892/or.2014.3585.

[73]Chihara T, Shimpo K, Beppu H, Yamamoto N, Kaneko T, Wakamatsu K, et al. Effects of aloe-emodin and emodin on proliferation of the MKN45 human gastric cancer cell line. Asian Pac $\mathrm{J}$ Cancer Prev. 2015;16(9):3887-3891. doi: 10.7314/APJCP.2015.16.9.3887.

[74]Liu A, Chen H, Wei W, Ye S, Liao W, Gong J, et al. Antiproliferative and antimetastatic effects of emodin on human pancreatic cancer. Oncol Rep. 2011;26(1):81-89

[75]Qu W, Wang Y, Wu Q, Liu J, Hao D. Emodin inhibits HMGB1-induced tumor angiogenesis in human osteosarcoma by regulating SIRT1. Int J Clin Exp Med. 2015;8(9):15054-15064.

[76]Gao, Yuan et al. 2014. "Shuang-Huang-Lian Exerts Anti-Inflammatory and Anti-Oxidative Activities in Lipopolysaccharide-Stimulated Murine Alveolar Macrophages." Phytomedicine: international journal of phytotherapy and phytopharmacology 21(4): 461-69.

[77]Gu, Naibing et al. 2012. "Shuanghuanglian Injection Downregulates Nuclear Factor-Kappa B Expression in Mice with Viral Encephalitis." Neural regeneration research 7(33): 2592-99.

[78]Ma, Qinhai et al. 2017. "Comparative Study on the Antivirus Activity of Shuang-Huang-Lian Injectable Powder and Its Bioactive Compound Mixture against Human Adenovirus III In Vitro." Viruses 9(4).

[79]Tang, Yuling et al. 2018. "Antiviral Effects of Shuanghuanglian Injection Powder against Influenza A Virus H5N1 in Vitro and in Vivo." Microbial pathogenesis 121: 318-24.

\section{Tables}

Table 1 Top 10 biological processes (BP), cell composition (CC), and molecular function (MF) in GO analysis 


\section{ONTOLOGY Description}

\begin{tabular}{|c|c|}
\hline $\mathrm{BP}$ & response to steroid hormone \\
\hline $\mathrm{BP}$ & response to ketone \\
\hline $\mathrm{BP}$ & reproductive structure development \\
\hline $\mathrm{BP}$ & reproductive system development \\
\hline $\mathrm{BP}$ & response to toxic substance \\
\hline $\mathrm{BP}$ & response to lipopolysaccharide \\
\hline $\mathrm{BP}$ & response to molecule of bacterial origin \\
\hline $\mathrm{BP}$ & aging \\
\hline $\mathrm{BP}$ & epithelial cell proliferation \\
\hline $\mathrm{BP}$ & regulation of epithelial cell proliferation \\
\hline $\mathrm{CC}$ & membrane raft \\
\hline $\mathrm{CC}$ & membrane microdomain \\
\hline $\mathrm{CC}$ & membrane region \\
\hline $\mathrm{CC}$ & caveola \\
\hline $\mathrm{CC}$ & plasma membrane raft \\
\hline $\mathrm{CC}$ & presynaptic membrane \\
\hline $\mathrm{CC}$ & integral component of presynaptic membrane \\
\hline $\mathrm{CC}$ & integral component of postsynaptic membrane \\
\hline $\mathrm{CC}$ & intrinsic component of postsynaptic membrane \\
\hline $\mathrm{CC}$ & intrinsic component of presynaptic membrane \\
\hline $\mathrm{MF}$ & nuclear receptor activity \\
\hline $\mathrm{MF}$ & transcription factor activity, direct ligand regulated sequence-specific DNA binding \\
\hline $\mathrm{MF}$ & steroid hormone receptor activity \\
\hline $\mathrm{MF}$ & G protein-coupled amine receptor activity \\
\hline $\mathrm{MF}$ & steroid binding \\
\hline $\mathrm{MF}$ & G protein-coupled neurotransmitter receptor activity \\
\hline $\mathrm{MF}$ & protein heterodimerization activity \\
\hline $\mathrm{MF}$ & heme binding \\
\hline $\mathrm{MF}$ & DNA-binding transcription activator activity, RNA polymerase II-specific \\
\hline $\mathrm{MF}$ & tetrapyrrole binding \\
\hline
\end{tabular}

Table 2 Parameters related to bar graphs of signal pathways enriched by drug target genes acting on diseases 


\begin{tabular}{|c|c|c|}
\hline Kaposi sarcoma-associated herpesvirus infection & $22 / 105$ & $1.71 \mathrm{E}-13$ \\
\hline Human cytomegalovirus infection & $22 / 105$ & $6.35 \mathrm{E}-12$ \\
\hline Hepatitis B & $19 / 105$ & $1.06 \mathrm{E}-11$ \\
\hline Small cell lung cancer & $15 / 105$ & $2.74 \mathrm{E}-11$ \\
\hline Colorectal cancer & $14 / 105$ & $1.46 \mathrm{E}-10$ \\
\hline Proteoglycans in cancer & $19 / 105$ & $3.86 \mathrm{E}-10$ \\
\hline IL-17 signaling pathway & $14 / 105$ & $3.86 \mathrm{E}-10$ \\
\hline Fluid shear stress and atherosclerosis & $16 / 105$ & $6.11 \mathrm{E}-10$ \\
\hline Estrogen signaling pathway & $15 / 105$ & $5.06 \mathrm{E}-09$ \\
\hline Measles & $15 / 105$ & 5.06E-09 \\
\hline p53 signaling pathway & $11 / 105$ & $3.26 \mathrm{E}-08$ \\
\hline TNF signaling pathway & $13 / 105$ & $3.26 \mathrm{E}-08$ \\
\hline Apoptosis & $14 / 105$ & 3.42E-08 \\
\hline Thyroid hormone signaling pathway & $13 / 105$ & $6.02 \mathrm{E}-08$ \\
\hline Endocrine resistance & $12 / 105$ & $6.24 \mathrm{E}-08$ \\
\hline Apoptosis - multiple species & $8 / 105$ & 7.33E-08 \\
\hline Hepatitis C & $14 / 105$ & $1.46 \mathrm{E}-07$ \\
\hline Neuroactive ligand-receptor interaction & $20 / 105$ & $1.46 \mathrm{E}-07$ \\
\hline Human immunodeficiency virus 1 infection & $16 / 105$ & 1.48E-07 \\
\hline
\end{tabular}

Table 3 Parameters related to bubble maps of signal pathways enriched by drug target genes acting on diseases 


\begin{tabular}{cccc}
\hline Human cytomegalovirus infection & 22 & 0.21 & $6.35 \mathrm{E}-12$ \\
& & & \\
\hline Neuroactive ligand-receptor interaction & 20 & 0.19 & $1.46 \mathrm{E}-07$
\end{tabular}

$19 \quad 0.18 \quad 4.09 \mathrm{E}-15$

\begin{tabular}{cccc}
\hline Hepatitis B & 19 & 0.18 & $1.06 \mathrm{E}-11$ \\
\hline Proteoglycans in cancer & 19 & 0.18 & $3.86 \mathrm{E}-10$ \\
\hline Fluid shear stress and atherosclerosis & 16 & 0.15 & $6.11 \mathrm{E}-10$ \\
\hline Human immunodeficiency virus 1 infection & 16 & 0.15 & $1.48 \mathrm{E}-07$
\end{tabular}

\begin{tabular}{cccc}
\hline Small cell lung cancer & 15 & 0.14 & $2.74 \mathrm{E}-11$ \\
\hline Estrogen signaling pathway & 15 & 0.14 & $5.06 \mathrm{E}-09$ \\
Measles & 15 & 0.14 & $5.06 \mathrm{E}-09$ \\
\hline Colorectal cancer & 14 & 0.13 & $1.46 \mathrm{E}-10$ \\
\hline IL-17 signaling pathway & 14 & 0.13 & $3.86 \mathrm{E}-10$ \\
\hline Apoptosis & 14 & 0.13 & $3.42 \mathrm{E}-08$ \\
\hline Hepatitis C & 14 & 0.13 & $1.46 \mathrm{E}-07$ \\
\hline TNF signaling pathway & 13 & 0.12 & $3.26 \mathrm{E}-08$ \\
\hline Thyroid hormone signaling pathway & 13 & 0.12 & $6.02 \mathrm{E}-08$ \\
\hline Endocrine resistance & 12 & 0.11 & $6.24 \mathrm{E}-08$ \\
\hline p53 signaling pathway & 11 & 0.10 & $3.26 \mathrm{E}-08$ \\
\hline Apoptosis - multiple species & & 0.08 & $7.33 \mathrm{E}-08$ \\
\hline
\end{tabular}

\section{Figures}




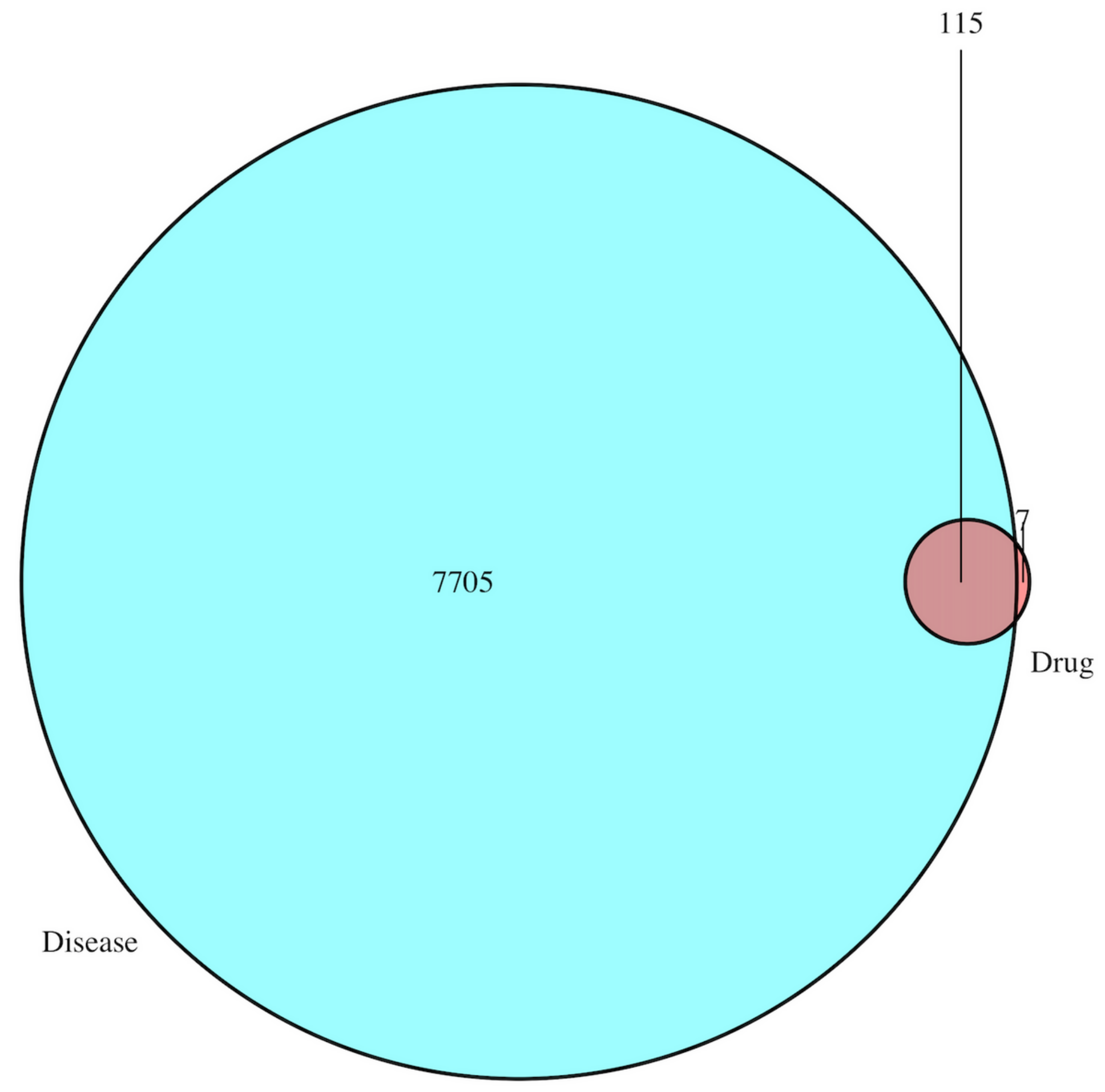

Figure 1

venn diagram of Shuanghuanglian related active ingredient gene target and SARS disease gene target 


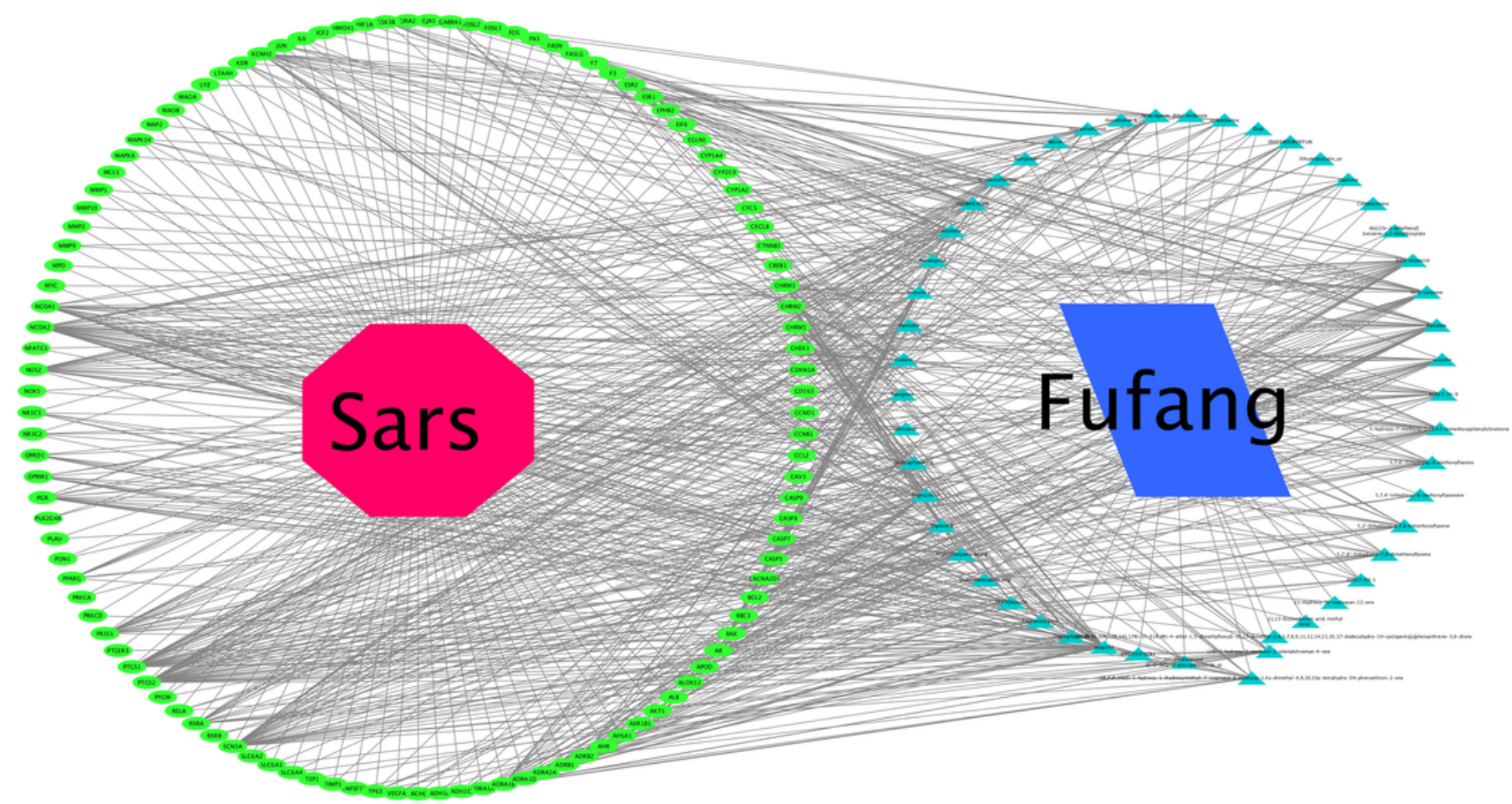

Figure 2

Shuanghuanglian active ingredient-drug gene target-disease gene target interaction network 

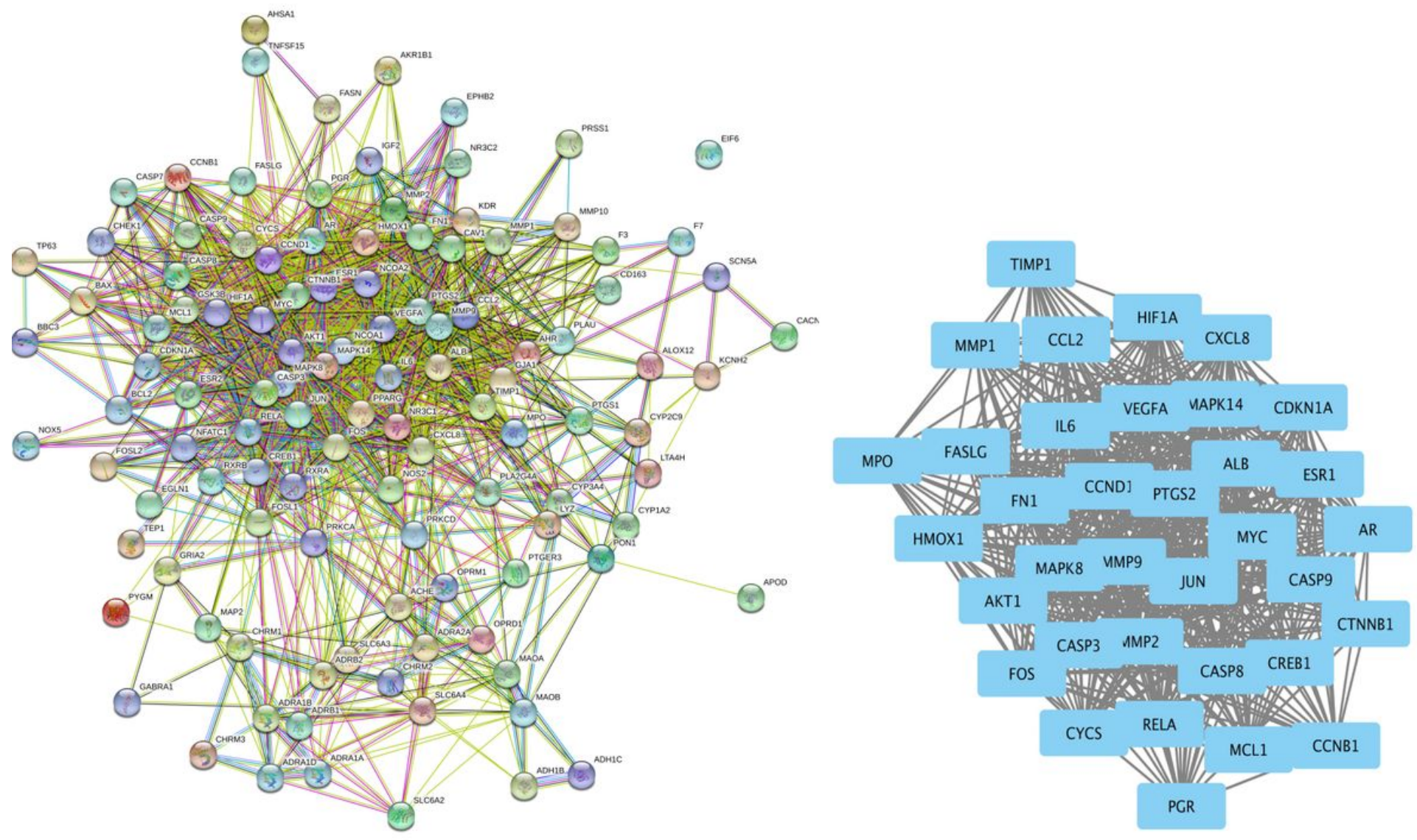

$\mathrm{C}$

$\mathrm{D}$

$\mathrm{E}$

$\mathrm{F}$
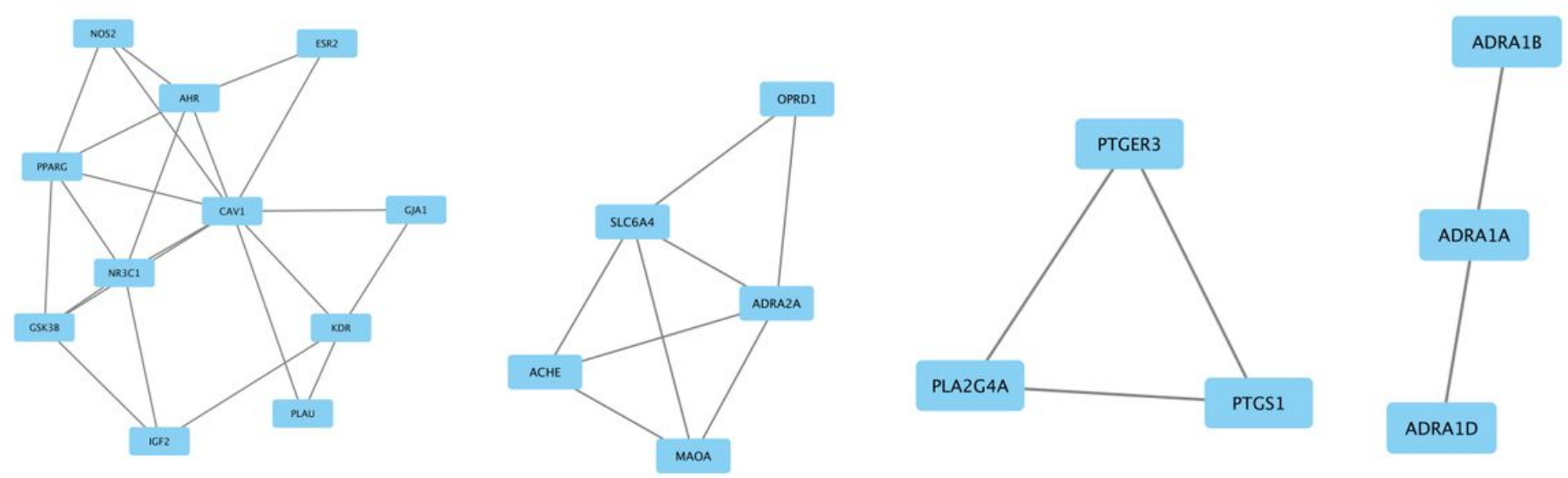

Figure 3

3A: protein interaction network for drug gene targets acting on diseases $₫ 3 \mathrm{~B}-\mathrm{F}$ : 5 cluster interaction networks 

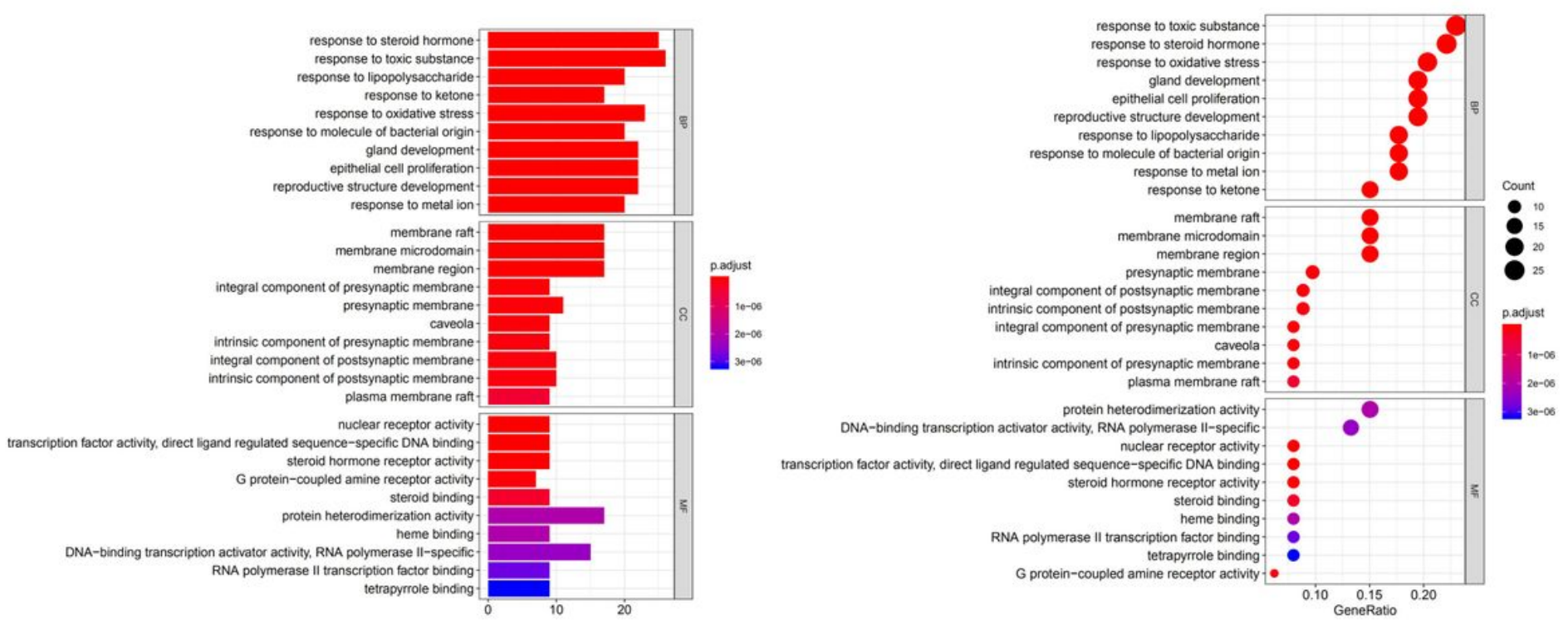

B
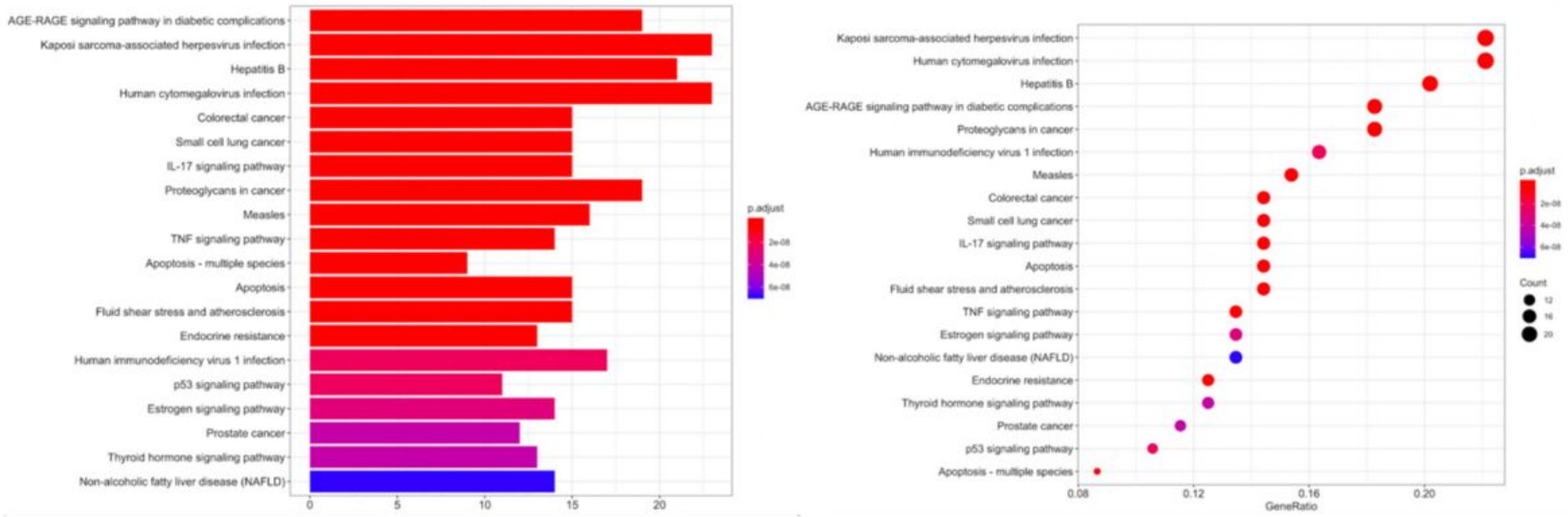

Figure 4

4A: Gene enrichment analysis of drug gene targets acting on diseases 4B: Signal pathway analysis of drug gene targets acting on diseases 